\title{
On rate improvements and power saving with opportunistic relaying in home power line networks
}

\author{
Salvatore D'Alessandro and Andrea M Tonello*
}

\begin{abstract}
In this article, we investigate the use of half duplex time division relay protocols to possibly provide achievable rate improvements, power saving, and coverage extension in home power line communication (PLC) networks. We consider a network where the communication between the source and the destination nodes follows an opportunistic protocol, namely, the relay is used whenever it allows with respect to the direct transmission: (a) for achievable rate improvements, under a power spectral density (PSD) mask constraint; or (b) for power saving, under a PSD mask and a rate target constraint. Opportunistic decode and forward (ODF), and opportunistic amplify and forward (OAF) are considered. At the physical layer, we assume the use of multi-carrier modulation. Under these assumptions, we find the optimal resource allocation, namely, the optimal power and time slot allocation, between the source and the relay nodes that maximizes the achievable rate, or minimizes the total transmitted power for both ODF and OAF. For the power minimization problem of ODF, we show that the joint problem of power and time slot allocation of DF is very hard to be solved and thus implemented. Therefore, to reduce complexity, we propose a simplified algorithm that considers two convex sub-problems. Through numerical results, we show that its performance is very close to the optimum. Finally, since over in-home PLC networks, the relay can only be placed in accessible points of the network, i.e., in outlets, or in the main panel, or in derivation boxes, for each opportunistic protocol, we also investigate the influence on performances from the relay position. Results are obtained using both measured channel responses and generated ones; thanks to the use of a statistically representative simulator. They show that significant rate improvements and power savings can be obtained as a function of the relay position and network size.
\end{abstract}

Keywords: Relay, Multi-carrier modulation, Power allocation, Power line communication, Resource allocation

\section{Introduction}

Power saving is playing an important role in developments of advanced communication devices. For instance, the IEEE 802.3az Ethernet standard and the HomePlug Green PHY (GP) power line communication (PLC) specifications have been developed to specifically address this problem. Not only power saving, but also high transmission rate has to be granted, as for instance in multimedia applications such as high definition television or 3D virtual video games. It becomes therefore essential to consider advanced communication techniques such as multicarrier modulation with bit and power loading algorithms,

*Correspondence: tonello@uniud.it

WiPli Lab, Università di Udine, Udine, Italy cooperative communication algorithms, and cross-layer optimization.

In this study, we investigate the use of cooperative half duplex time division relay protocols to possibly provide power savings, achievable rate improvements, and coverage extension to the in-home PLC networks whose communication devices adopt multi-carrier modulation at the physical (PHY) layer, i.e., orthogonal frequency division multiplexing (OFDM) [1].

The problem of resource allocation in relay networks has thoroughly been treated in the wireless literature. In the following, a number of relevant articles on the topic is reported.

The optimal power and time slot allocation for capacity maximization over flat Rayleigh fading relay channels has been considered in [2-4]. The case of power allocation

\section{Springer}

(C) 2012 D'Alessandro and Tonello; licensee Springer. This is an Open Access article distributed under the terms of the Creative Commons Attribution License (http://creativecommons.org/licenses/by/2.0), which permits unrestricted use, distribution, and reproduction in any medium, provided the original work is properly cited. 
for capacity maximization of single hop parallel Gaussian relay channels (e.g., OFDM systems) under a total power constraint has been treated in [5-10]. In particular, in [5], the authors found a sub-optimal power allocation considering half duplex amplify and forward (AF) and a total power constraint at the source and relay nodes. The optimal solution to the previous problem has been found in [6]. In [7], the authors found the optimal power allocation for half duplex AF and decode and forward (DF) under a source plus relay power constraint of each OFDM sub-channel. Both previous articles assume that the destination node is not directly reachable from the source. In [8], the power allocation for full-duplex [2] DF under a total power constraint (source and relay) is considered. The optimal power allocation for the hybrid use of AF, DF, and direct link transmission is computed in [9], under a source plus relay power constraint of each sub-channel. In [10], the authors found the optimal power allocation for full and half duplex DF under a total power constraint at the source and destination nodes. Cooperation with multiple relays, or relays with multiple antennas have extensively been treated in the wireless literature, cf. e.g., $[11,12]$ and references therein.

In this article, we consider the specific and peculiar application of relaying in the in-home PLC scenario. In contrast to the wireless case, the use of relaying for PLC has not deeply been investigated yet. In [13], the authors considered large ad-hoc PLC networks where the source and destination nodes are far apart so that they cannot directly communicate. In this circumstance, it is shown that the application of distributed space-time coding to multiple relays can be used to improve the network performances in terms of required transmit power and multihop delay w.r.t. some alternative solutions, e.g., simultaneous simple retransmission. In [14], the authors proposed practical sub-channel and power allocation algorithms for a two-hop DF relay scheme to improve the achievable rate of an orthogonal frequency division multiple access PLC in-home network. The numerical results were obtained using a small number of measured channels and assuming a total power constraint at each network node. Some results are reported in [15], in terms of achievable rate for AF and DF schemes over PLC channels. The network nodes employ OFDM at the PHY layer and the power is equally distributed among the used OFDM sub-channels. The channel model used, although based on transmission line theory, does not consider the peculiarities of inhome wiring topologies. Numerical results show that the use of half duplex single relay schemes leads to marginal improvements of the achievable rate w.r.t. the direct transmission (DT). However, this result is only partially true, since the opportunistic use of the relay was not considered and the dependency on the relay position was not thoroughly investigated. Finally, in [16], the authors extended the work by considering the effect of channel estimation errors.

In this study, we consider a network whose nodes have a PHY layer based on OFDM and where the communication between the source and the destination nodes follows an opportunistic protocol, namely, the relay is used whenever it allows (w.r.t. the DT): (a) for achievable rate improvements under a PSD mask constraint, or (b) for power saving under a PSD mask and a rate target constraint. Opportunistic DF (ODF), and opportunistic AF (OAF) are considered. As it is typically required by state-of-the-art communication standard, e.g., the wireless IEEE 802.11 standard, the power line IEEE P1901 standard, and the twisted pair xDSL standard, we assume that the signal transmitted by the network nodes has to satisfy a power spectral density (PSD) mask.

Under these assumptions and Gaussian noise model, we find the optimal resource allocation, namely, the optimal power and time slot allocation, at the source and relay nodes that maximizes achievable rate, or minimizes the total transmitted power for both ODF and OAF.

This study brings two main new contributions w.r.t. the ones presented in the literature. The first contribution regards the analysis of multi-carrier power allocation algorithms for half duplex time division two-hop relay networks where the power transmitted by each network node is constrained by a PSD mask, rather than a total power constraint. In particular, for the achievable rate maximization problem of ODF, we find the optimal power and time slot allocation. Whatever the time slot duration is, the power allocation that maximizes the DF achievable rate is equal to the one that satisfies the PSD mask. Consequently, we show that the optimal time slot duration is found by the intersection of two linear functions. For the power minimization problem of ODF, we show that it is very hard to solve the problem of joint power and time slot allocation. Therefore, to reduce complexity, we propose a simplified algorithm that considers two convex sub-problems. Through numerical results, we show that its performance is very close to the optimal one.

The second-and of practical relevance-contribution is related to the application of relaying to the specific and peculiar in-home PLC scenario. Differently from the wireless case, where the relay can be placed wherever between the source and the destination nodes, in in-home PLC networks, the relay can only be placed in accessible points of the network, i.e., in the outlets or in the main panel (MP) or, in principle, in the derivation boxes. To investigate the benefits that can be obtained by the use of the relay placed in the network outlets, we make use of experimental measured channels. Furthermore, to infer the effect on performance in a broader set of in-home topologies, with the placement of the relay in different accessible points, 
we show results using a PLC channel generator based on a statistically topology model and transmission line theory $[17,18]$. The topology model is representative of Italian and EU scenarios. Both achievable rate improvements and power saving are investigated. Furthermore, we also consider OAF to benchmark results with a simple relay scheme. Finally, we note that some preliminary results of this study have been presented in [19].

The remainder of the article is as follows. In "PLC system model" section, we describe the PLC system model. Then, in "Opportunistic Decode and Forward" and "Opportunistic Amplify and Forward" sections, we, respectively, consider the resource allocation problem of ODF and OAF. "Numerical results" section discusses numerical results. Finally, conclusions follow.

\section{PLC system model}

We consider an in-home PLC network where the communication between the source $(S)$ and the destination (D) nodes exploits the use of a relay (R) (Figure 1). In particular, we consider that the communication between the source and the destination nodes follows an opportunistic cooperative protocol, namely, the relay is used whenever it allows, according to the goal, for rate improvements or for power saving w.r.t. the DT. The multiplexing between the source and the relay nodes is accomplished via time division multiple access. The time is divided into frames of duration $T_{f}$, and each frame is divided into two time slots whose durations are $\tau$ and $T_{f}-\tau$. When the relay is used, the source transmits its data to the relay and destination nodes during the first slot-although it is possible that the source cannot directly reach the destinationwhereas, during the second slot, the source is silent and the relay transmits the received data to the destination according to the adopted opportunistic cooperative protocol, i.e., ODF or OAF. When ODF is used, the relay decodes, re-encodes and forwards the received data using an independent codebook. Whereas, in OAF, the relay only amplifies and forwards the data (see Figure 2).
At the PHY layer, we assume OFDM with $M$ subchannels. The channel frequency response between each pair of nodes is denoted as $H_{x, y}^{(k)}$, where the subscripts $x$ and $y$ denote the pairs $\{\mathrm{S}, \mathrm{R}\},\{\mathrm{S}, \mathrm{D}\}$, or $\{\mathrm{R}, \mathrm{D}\}$, and $k$ is the sub-channel index, i.e., $k \in \mathbb{K}_{\text {on }}$, where $\mathbb{K}_{\text {on }} \subseteq\{0, \ldots, M-$ 1 \} is the sub-set of used (switched on) sub-channels that allows for satisfying a PSD mask with notches, as it is the case in broadband PLC systems [20]. Therefore, the received signal in the $k$-th sub-channel of the $y$-th node reads

$$
z_{y}^{(k)}=a_{x}^{(k)} H_{x, y}^{(k)}+w_{y}^{(k)},\{x, y\} \in\{\{S, D\} ;\{S, R\} ;\{R, D\}\}, k \in \mathbb{K}_{\mathrm{on}},
$$

where $a_{x}^{(k)}$ is the symbol transmitted by node $x$ in subchannel $k$ using DT, DF, or AF modes, while $w_{y}^{(k)}$ is the background noise. We assume the noise and the transmitted symbols to be i.i.d. and drawn from normal Gaussian distributions with zero mean and power $P_{w, y}^{(k)}$ and $P_{x \text {,mode' }}^{(k)}$, respectively. Throughout this study, we assume that the application of a PSD mask constraint for the signal transmitted by the network nodes. Furthermore, in order to simplify the notation, we assume the PSD to be constant over the sub-channels, i.e., $P_{x, \text { mode }}^{(k)} \leq \bar{P} \forall k \in \mathbb{K}_{\mathrm{on}}, x \in$ $\{S ; R\}$, and mode $\in\{D T ; D F ; A F\}$. We highlight that all the power allocation algorithms that will be studied in this article are also valid when a more general non-constant PSD is considered.

\section{In-home power line network topology}

As discussed in the Introduction, we are interested to see whether achievable rate improvements, power savings, and coverage extension are attainable through the use of ODF and OAF. To this end, in the following, we describe a typical PLC network topology, which allows for understanding where relays can be placed, and it highlights the differences with the wireless context. It is representative of the majority of Italian and EU residential wiring structures [21].

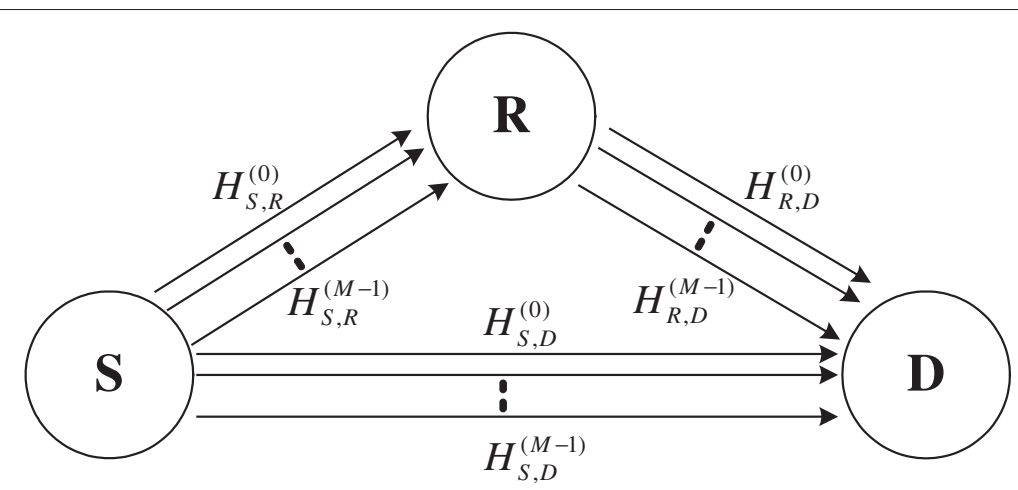

Figure 1 Cooperative relay system model. 


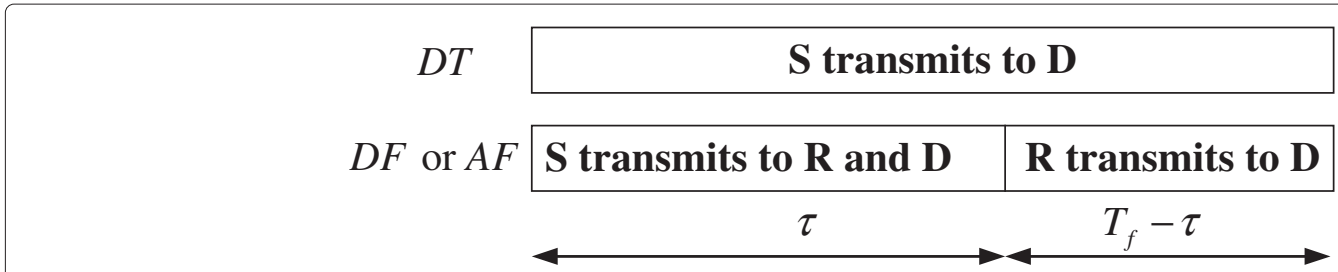

Figure 2 DT, DF, and AF modes and corresponding time slots allocation.

In particular, it is characterized by a wiring topology composed of two layers. As it is shown in Figure 3, the outlets are placed at the bottom layer and are grouped and fed by the same "super node", which is referred to as derivation box. All the outlets fed by the same derivation box are nearby placed. Therefore, the location plan is divided into elements denoted as "clusters" that contain a derivation box with the associated outlets. Each cluster represents a room or a small number of nearby rooms. Different clusters are usually interconnected through their derivation boxes with dedicated cables. This set of interconnections forms the second layer of the topology. We refer to the channels that connect pair of outlets belonging to the same cluster as intracluster channels. Whereas, the channels associated to pairs of outlets that belong to different clusters are referred to as intercluster channels. An intercluster channel example is shown in Figure 3.

The MP plays a special role inasmuch it connects the home network with the energy supplier network through circuit breakers (CBs). We distinguish two cases. The first case, which we refer to as single-sub-topology networks, is when a single CB feeds all the derivation boxes of the home network. The second case, which we refer to as multi-sub-topology networks, is when many subtopologies, each comprising a group of derivation boxes, have their own electrical circuit that is interconnected at the MP through a CB. The latter case can be, for instance, representative of a multi-floor house, where each floor is a sub-topology. In Figure 3, we report an example of a two-sub-topology network.

Now, we consider the communication between source and destination nodes with the help of a relay. In particular, we consider source-destination channels defined between pair of outlets that do not belong to the same cluster, i.e., intercluster channels. As shown in [17], these channels experience higher attenuations than intracluster channels. Thus, they can benefit more from the presence of a relay. Clearly, these benefits are also dependent on the relay location. To this end, for single-sub-topology networks, the relay can be strategically placed in the following manners.

- Outlet relay arrangement (ORA). The relay is placed in a randomly selected network outlet.

- MP single sub-topology (MPS). The relay is placed immediately after the CB of the MP.

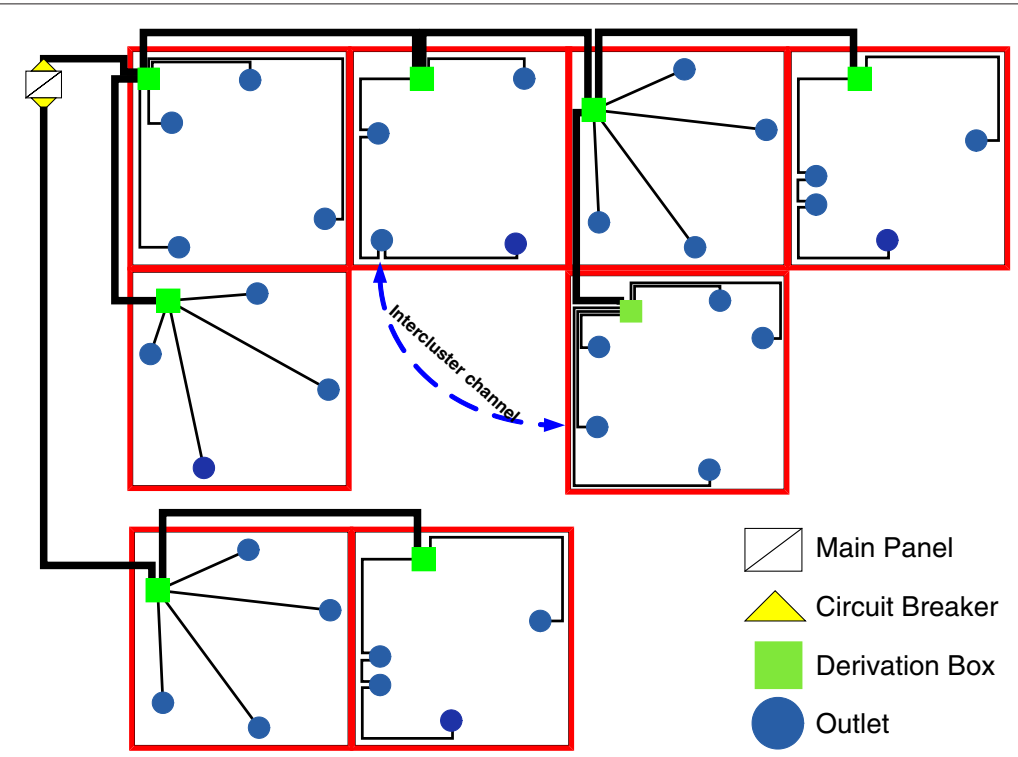

Figure 3 Network architecture with two sub-topologies. 
- Random derivation box (RDB). The relay is placed in a randomly selected network derivation box. In general, derivation boxes are accessible although they do not have an already installed outlet. Thus, a relay can be installed inside the box or nearby.

- Backbone derivation box (BDB). The relay is located in a randomly selected derivation box that belongs to the backbone between the source and the destination nodes. Note that, for intercluster channels, the source and destination nodes are at least divided by the source and destination derivation boxes.

- Source derivation box (SDB). The relay is located in the derivation box that feeds the source node. Note that for intercluster channels the path between source and destination includes at least the derivation box that feeds the source and the one that feeds the destination.

- Destination derivation box (DDB). The relay is located in the derivation box that feeds the destination node.

When we consider multi-sub-topology networks, we assume that the source and the destination nodes are located into two different sub-topologies. In such a case, we can consider the following strategical configurations for the relay.

- MP multi-sub-topology (MPM). The relay is located between the CBs that feed the sub-topologies.

- ORA source sub-topology (ORAS). The relay is located in a randomly selected outlet belonging to the same sub-topology of the source node.

- ORA destination sub-topology (ORAD). The relay is located in a randomly selected outlet belonging to the same sub-topology of the destination node.

\section{Opportunistic Decode and Forward}

In ODF, the source node sends data to the destination node according to two modes, DT or DF. Assuming a frame of normalized duration $T_{f}=1$, we can compute the achievable rate of ODF as [3]

$$
C_{\mathrm{ODF}}(\tau)=\max \left\{C_{\mathrm{DT}}, C_{\mathrm{DF}}(\tau)\right\}
$$

where $C_{\mathrm{DT}}$ and $C_{\mathrm{DF}}(\tau)$, respectively, denote the capacity of DT and the achievable rate of DF [2]. These are given by

$$
\begin{aligned}
C_{\mathrm{DT}} & =C_{S, D} \\
C_{\mathrm{DF}}(\tau) & =\min \{\underbrace{\tau C_{S, R}}_{f_{1}\left(\tau, \mathbf{P}_{\mathbf{S}, \mathbf{D F}}\right)}, \underbrace{\tau C_{S, D}+(1-\tau) C_{R, D}}_{f_{2}\left(\tau, \mathbf{P}_{\mathbf{S}, \mathbf{D F}}, \mathbf{P}_{\mathbf{R}, \mathbf{D F}}\right)}\} .
\end{aligned}
$$

In (3) and (4), $C_{S, D}, C_{S, R}$ and $C_{R, D}$ denote the capacities of the links $\mathrm{S}-\mathrm{D}, \mathrm{S}-\mathrm{R}$, and $\mathrm{R}-\mathrm{D}$, respectively. Assuming the system model of "PLC system model" section, they are given by [22]

$$
\begin{aligned}
C_{x, y}= & \frac{1}{M T} \sum_{k \in \mathbb{K}_{\mathrm{on}}} \log _{2}\left(1+S N R_{x, y}^{(k)}\right), \\
& \{x, y\} \in\{\{S, D\} ;\{S, R\} ;\{R, D\}\},
\end{aligned}
$$

where

$$
S N R_{x, y}^{(k)}=P_{x, \text { mode }}^{(k)} \frac{\left|H_{x, y}^{(k)}\right|^{2}}{P_{w, y}^{(k)}}=P_{x, \text { mode }}^{(k)} \eta_{x, y}^{(k)},
$$

is the signal-to-noise ratio (SNR) in sub-channel $k$ for the link $x-y, T$ is the sampling period, and $\eta_{x, y}^{(k)}$ denotes the normalized SNR for the link $x-y$ in sub-channel $k$. Furthermore, in (4), $\mathbf{P}_{\mathbf{S}, \mathbf{D F}}$ and $\mathbf{P}_{\mathbf{R}, \mathbf{D F}}$ denote the vectors (with $\left|\mathbb{K}_{\text {on }}\right|$ elements) of the sub-channel powers at the source and at the relay node, respectively. As it will be clear in the following, it is convenient to express the arguments of the minimization in (4) through the functions $f_{1}$ and $f_{2}$.

To simplify the notation, in (5), we do not explicitly show the dependance of the capacity from the transmitted power distribution, which will be done if needed in the following. We notice that an SNR gap can be used in (5) to take into account that practical coding and modulation schemes are used, e.g., the HPAV broadband PLC system employs turbo codes that allow for having an SNR gap of less than $3 \mathrm{~dB}$ versus capacity (5) [23]. Furthermore, in (5), we have implicitly assumed perfect channel state information, this is because in this study we want to investigate theoretical performances. However, in this respect, we notice that PLC channels can be considered time invariant over the duration of several OFDM symbols, which allows accurate SNR estimation. It should also be noted that our analysis is in terms of achievable rate that corresponds to the definition of delay-limited capacity according to $[3,24]$. This capacity formulation is appropriate especially for delay sensitive applications as voice and video where long delays cannot be tolerated. In this respect, sufficiently long codes can achieve the instantaneous capacity defined in (4) and (5) since the PLC channel can be assumed constant for a long period of time. Channel variations are due to topology changes. In practice moderate long codes, that introduce tolerable delay, should come close to the theoretical limit.

From (2)-(4), it is interesting to note that a necessary condition not to use the direct link is $C_{S, D}<$ $\min \left\{C_{S, R}, C_{R, D}\right\}$. In the remaining cases, to see whether the communication follows the DT or the DF mode, we need to compute $C_{\mathrm{DT}}, C_{\mathrm{DF}}(\tau)$ and compare them as in (2) to determine the largest. We also note that (2)-(4) already take into account the case in which the destination cannot listen to the source, i.e., $\left|H_{S, D}^{(k)}\right|=0 \forall k \in \mathbb{K}_{\mathrm{on}}$.

In "Rate improvements with ODF" and "Power saving with ODF" sections, we will, respectively, deal with the 
power allocation for achievable rate improvements and power saving of ODF.

\section{Rate improvements with ODF}

From (2), we note that the achievable rate of ODF is a function of both the transmitted power distribution and the time slot allocation. In order to maximize it, when the DT is used, we only need to optimally allocate the power among the sub-channels of the source node. To the contrary, when the DF mode is used, we need to optimally allocate the power and the time slot of the source and relay.

Assuming that the network nodes have to satisfy a PSD mask constraint, it is known that the sub-channel power allocation that maximizes the capacity for a point-to-point communication corresponds to the one given by the PSD constraint itself [25]. This can simply be proven by observing that the capacity formula (5) monotonically increases with the sub-channel allocated power. Therefore, for both ODF transmission modes, we set $P_{x \text {,mode }}^{(k)}=\bar{P}$, with $x \in$ $\{S, R\}$, and $k \in \mathbb{K}_{\mathrm{on}}$. Now, to maximize the ODF achievable rate (2), we only need to compute the optimal time slot duration that can be found maximizing (4), i.e.,

$$
\tau_{m r}^{*}=\underset{\tau \in[0,1]}{\operatorname{argmax}}\left\{C_{\mathrm{DF}}(\tau)\right\},
$$

where we have used the subscripts $m r$ to indicate that $\tau_{m r}^{*}$ is the time slot duration that maximizes the achievable rate. To solve (7), we observe that once the power transmitted by the source and the destination nodes is set, the arguments of the minimization in (4) are linear functions of $\tau$. Assuming that $C_{S, R} \geq C_{S, D}$, the optimal time slot duration $(0 \leq \tau \leq 1)$ is given by the intersection $f_{1}\left(\tau_{m r}^{*}, \mathbf{P}_{\mathbf{S}, \mathbf{D F}}\right)=f_{2}\left(\tau_{m r}^{*}, \mathbf{P}_{\mathbf{S}, \mathbf{D F}}, \mathbf{P}_{\mathbf{R}, \mathbf{D F}}\right)$, with $P_{S, D F}^{(k)}=$ $P_{R, D F}^{(k)}=\bar{P} \forall k \in \mathbb{K}_{\mathrm{on}}$.

\section{Power saving with ODF}

In this section, we consider the use of ODF for power saving and coverage extension. As discussed in the previous section, in ODF the relay is used when the DF achievable rate is higher than that of DT. Now, let us suppose that the relay is used and we want to achieve a given target rate under a PSD constraint. Then, we can have three cases. The first case is when the target rate is reachable either using DT or DF. In such a case, since the DF achievable rate is higher than that of DT, the amount of power saved lowering the rate of DF to the target value will be higher than that saved lowering the rate of DT to the target value. The second case is when only the DT rate is lower than the target rate. In this case, the use of the relay can increase the network coverage. The third case is when the achievable rate of both modes is lower than the target rate so that the use of the relay increases the achieved rate possibly toward the target.
To compute the power necessary by ODF to achieve a target rate $R$ when the communication is subject to a PSD constraint, we can solve the problem

$$
P_{\mathrm{ODF}}=\min \left\{P_{\mathrm{DT}}, P_{\mathrm{DF}}\right\},
$$

where $P_{\mathrm{DT}}$ and $P_{\mathrm{DF}}$, respectively, denote the minimum power required by the DT and DF modes to achieve a rate $R$ under a PSD constraint. Therefore, $P_{\mathrm{DT}}$ is the solution to the problem

$$
P_{\mathrm{DT}}=\min \sum_{k \in \mathbb{K}_{\mathrm{on}}} P_{S, \mathrm{DT}}^{(k)}
$$

s.t. $C_{S, D}=R$,

$$
0 \leq P_{S, \mathrm{DT}}^{(k)} \leq \bar{P} \quad \forall \quad k \in \mathbb{K}_{\mathrm{on}},
$$

while $P_{\mathrm{DF}}$ is the solution to the problem

$$
\begin{array}{ll} 
& P_{\mathrm{DF}}=\min \sum_{k \in \mathbb{K}_{\mathrm{on}}} \tau P_{S, \mathrm{DF}}^{(k)}+(1-\tau) P_{R, \mathrm{DF}}^{(k)} \\
\text { s.t. } & C_{\mathrm{DF}}(\tau)=\min \left\{\tau C_{S, R}, \tau C_{S, D}+(1-\tau) C_{R, D}\right\}=R, \\
& 0 \leq \tau \leq 1, \\
& 0 \leq P_{S, \mathrm{DF}}^{(k)} \leq \bar{P}, \\
& 0 \leq P_{R, \mathrm{DF}}^{(k)} \leq \bar{P}, \quad \forall \quad k \in \mathbb{K}_{\mathrm{on}} .
\end{array}
$$

Starting from (9), we note that its objective and its inequality constraint functions are convex, but its equality constraint is not an affine function. Therefore, (9) is not in general a convex problem ([26], pp. 136-137). Nevertheless, we note that the equivalent problem (see [26], p. 67) for the definition of equivalent problems), obtained considering the change of variables $P_{S, \mathrm{DT}}^{(k)}=\left(2^{b_{S, \mathrm{DT}}^{(k)}}-\right.$ 1) $/ \eta_{S, D}^{(k)}$, where $b_{S, \mathrm{DT}}^{(k)}=\log _{2}\left(1+P_{S, \mathrm{DT}}^{(k)} \eta_{S, D}^{(k)}\right)$, is a convex optimization problem. The solution to the equivalent problem (assuming that it exists ${ }^{\mathrm{a}}$ ) it is well known and it can be found imposing the KKT conditions (cf. e.g., [25]). Hence, the solution to the original problem (9) can simply be found applying the inverse change of variables to the solution of the equivalent problem, and it is equal to

$$
P_{S, \mathrm{DT}}^{(k)}=P_{S, \mathrm{DT}}^{(k)}(v)=\left[v-1 / \eta_{S, D}^{(k)}\right]_{0}^{\bar{P}},
$$

where

$$
[x]_{a}^{b}= \begin{cases}b, & x \geq b \\ x, & a<x<b \\ a, & x \leq a,\end{cases}
$$

and $v$ is equal to the solution of the equality constraint of (9), i.e.,

$$
\sum_{k \in \mathbb{K}_{\mathrm{on}}} \log _{2}\left(1+P_{S, \mathrm{DT}}^{(k)}(v) \eta_{S, D}^{(k)}\right)=M R T .
$$

It is interesting to note that when a non-uniform PSD mask has to be satisfied, the solution to problem (9) remains the same as in (11), provided that the maximum 
allowable power in each sub-channel is set equal to the corresponding power constraint [25].

Now, turning our attention to problem (10), we note that it is more difficult to solve than problem (9) inasmuch its objective function is not in general convex. This can be proved observing that the Hessian associated to its objective function, for a given $k$, is neither semidefinite positive nor semidefinite negative, consequently, the Sylvester's criterion [27] does not give any information regarding convexity.

In "Optimal DF power allocation" section, we discuss the optimal solution to problem (10). Then, in "Simplified algorithm for DF power allocation" section, we propose a feasible algorithm that gives a sub-optimal solution.

\section{Optimal DF power allocation}

To find the optimal solution to (10), we observe that for a given value of $\tau$, problem (10) can be split into two convex sub-problems. The first sub-problem is obtained when $C_{\mathrm{DF}}(\tau)=\tau C_{S, R}$ and it reads

$$
P_{\mathrm{DF} 1}(\tau)=\min \sum_{k \in \mathbb{K}_{\mathrm{on}}} \tau P_{S, \mathrm{DF} 1}^{(k)}+(1-\tau) P_{R, \mathrm{DF} 1}^{(k)}
$$

s.t. $\quad \tau C_{S, R}=R$,

$$
\begin{aligned}
& \tau C_{S, D}+(1-\tau) C_{R, D} \geq R, \\
& 0 \leq P_{S, \mathrm{DF} 1}^{(k)} \leq \bar{P}, \\
& 0 \leq P_{R, \mathrm{DF} 1}^{(k)} \leq \bar{P}, \quad \forall \quad k \in \mathbb{K}_{\mathrm{on}} .
\end{aligned}
$$

In (14), we have used the subscript 1 to indicate the first sub-problem. Replacing in (14) $P_{S, \mathrm{DF} 1}^{(k)}=\left(2^{b_{S, \mathrm{DF} 1}^{(k)}}-1\right) / \eta_{S, R}^{(k)}$ and $P_{R, \mathrm{DF} 1}^{(k)}=\left(2^{b_{R, \mathrm{DF} 1}^{(k)}}-1\right) / \eta_{R, D}^{(k)}$, we obtain an equivalent convex optimization problem, whose variables are $b_{S, D F 1}^{(k)}$ and $b_{R, \mathrm{DF} 1}^{(k)}$, with $k \in \mathbb{K}_{\mathrm{on}}$.

The second sub-problem is obtained when $C_{\mathrm{DF}}(\tau)=$ $\tau C_{S, D}+(1-\tau) C_{R, D}$ and it reads

$$
\begin{array}{ll} 
& P_{\mathrm{DF} 2}(\tau)=\min \sum_{k \in \mathbb{K}_{\mathrm{on}}} \tau P_{S, \mathrm{DF} 2}^{(k)}+(1-\tau) P_{R, \mathrm{DF} 2}^{(k)} \\
\text { s.t. } & \tau C_{S, D}+(1-\tau) C_{R, D}=R, \\
& \tau C_{S, R} \geq R, \\
& 0 \leq P_{S, \mathrm{DF} 2}^{(k)} \leq \bar{P}, \\
& 0 \leq P_{R, \mathrm{DF} 2}^{(k)} \leq \bar{P}, \quad \forall \quad k \in \mathbb{K}_{\mathrm{on}} .
\end{array}
$$

Similar to (14), it is possible to obtain a problem that is equivalent to (15) and that is convex. It can be found with the change of variables $P_{S, \mathrm{DF} 2}^{(k)}=\left(2^{b_{S, \mathrm{DF} 2}^{(k)}}-1\right) / \eta_{S, D}^{(k)}$ and $P_{R, \mathrm{DF} 2}^{(k)}=\left(2^{b_{R, \mathrm{DF} 2}^{(k)}}-1\right) / \eta_{R, D}^{(k)}$.

Since both problems (14) and (15) can be reduced to convex problems, their solution ${ }^{\mathrm{a}}$ can be found imposing the KKT conditions to the equivalent problems ([26], pp. 243-244) and [28]. Although we here omit the proof, it can be shown that the solution to the KKT conditions requires an iterative procedure. Consequently, its complexity is not less than that of conventional methods used for solving inequality constrained minimization problems, e.g., the interior-point method ([26], chapter 11). Therefore, for the numerical results, we use the interior-point method. It should be noted that interior-point methods are in general complex, especially when the dimension of the problem is large.

Now, to compute the optimal DF power allocation, for each value of $\tau$, we can compute $P_{\mathrm{DF}}(\tau)=$ $\min \left\{P_{\mathrm{DF} 1}(\tau), P_{\mathrm{DF} 2}(\tau)\right\}$. Therefore, the optimal slot duration for minimum power can be found as

$$
\tau_{m p}^{*}=\min _{\tau \in[0,1]} P_{\mathrm{DF}}(\tau),
$$

and the minimum power required by $\mathrm{DF}$ to reach the rate $R$ is the one corresponding to $P_{\mathrm{DF}}^{*}=P_{\mathrm{DF}}\left(\tau_{m p}^{*}\right)$. Finally, we recall that to compute the power required by ODF to reach the rate $R$ under the PSD mask constraint, we can replace $P_{\mathrm{DF}}^{*}$ and (11) in (8).

\section{Simplified algorithm for DF power allocation}

To reduce complexity, we propose a simplified solution based on the following algorithm. We assume the optimal time slot duration $\tau_{m p}^{*}$ equal to the one computed in (7), i.e., $\tau_{m p}^{*}=\tau_{m r}^{*}$, where we have considered the achievable rate maximization under a PSD constraint. Furthermore, we impose the constraint that for $\tau_{m p}^{*}$ the arguments of the minimization in the second line of (10) are equal to $R$. Under these assumptions, (10) can be divided into two sub-problems where the first allows us to compute the power distribution of the source node independently from the power distribution of the relay node. Once we know the power distribution of the source, we can compute the power distribution of the relay solving the second sub-problem. Figure 4 illustrates the proposed algorithm. Practically, we first find the source power distribution that allows the linear function of $\tau, f_{1}\left(\tau, \mathbf{P}_{\mathbf{S}, \mathrm{DF}}\right)$, to pass through the origin of the axes and the point $p$ specified by the pair $\left\{\tau_{m p}^{*}, R\right\}$. Then, maintaining this power distribution at the source node, we compute the power distribution at the relay node that allows the function $f_{2}\left(\tau, \mathbf{P}_{\mathbf{S}, \mathbf{D F}}, \mathbf{P}_{\mathbf{R}, \mathbf{D F}}\right)$ to pass through the points $p$ and the one specified by the pair $\left\{1, C_{S, D}\right\}$.

In particular, the power distribution at the source can be computed solving the following sub-problem of (10)

$$
\begin{array}{ll} 
& P_{S, \mathrm{DF}}=\min \sum_{k \in \mathbb{K}_{\mathrm{on}}} P_{S, \mathrm{DF}}^{(k)} \\
\text { s.t. } & \tau_{m p}^{*} C_{S, R}=R, \\
& 0 \leq P_{S, \mathrm{DF}}^{(k)} \leq \bar{P} \quad \forall \quad k \in \mathbb{K}_{\mathrm{on}} .
\end{array}
$$




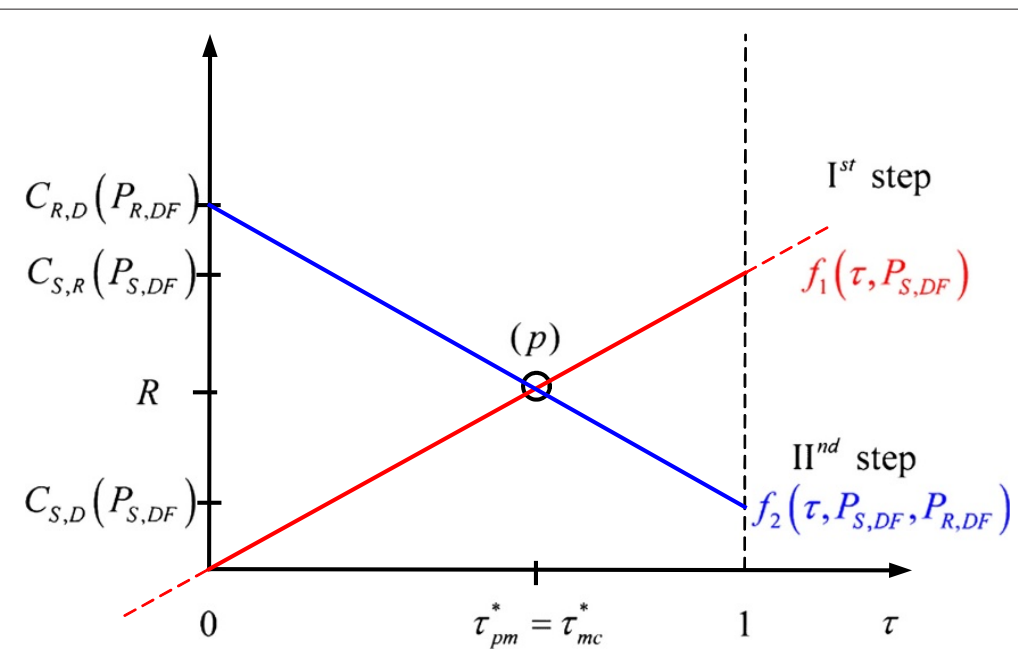

Figure 4 Illustration of the proposed power allocation algorithm to minimize the power required by DF to reach a target rate $R$.

Problem (17) can be solved similar to ${ }^{\mathrm{a}}(9)$. The solution is

$$
P_{S, \mathrm{DF}}^{(k)}=P_{S, \mathrm{DF}}^{(k)}(v)=\left[v-1 / \eta_{S, R}^{(k)}\right]_{0}^{\bar{P}},
$$

where $v$ is given by the solution of the second line of (17), i.e.,

$$
\sum_{k \in \mathbb{K}_{\mathrm{on}}} \log _{2}\left(1+P_{S, \mathrm{DF}}^{(k)}(\nu) \eta_{S, R}^{(k)}\right)=\frac{M R T}{\tau_{m p}^{*}}
$$

Once the "optimal" power distribution for the source node in DF mode has been computed, it can be used to compute the capacity of the $\mathrm{S}-\mathrm{D}$ link, namely $C_{S, D}$ in DF mode. Therefore, we can find the "optimal" power distribution for the relay node solving the following subproblem of (10)

$$
\begin{array}{ll} 
& P_{R, \mathrm{DF}}=\min \sum_{k \in \mathbb{K}_{\mathrm{on}}} P_{R, \mathrm{DF}}^{(k)} \\
\text { s.t. } & C_{R, D}=\hat{R}, \\
& 0 \leq P_{R, \mathrm{DF}}^{(k)} \leq \bar{P} \quad \forall \quad k \in \mathbb{K}_{\mathrm{on}} .
\end{array}
$$

whose solution $^{\mathrm{a}}$ is

$$
P_{R, \mathrm{DF}}^{(k)}=P_{R, \mathrm{DF}}^{(k)}(v)=\left[v-1 / \eta_{R, D}^{(k)}\right]_{0}^{\bar{P}},
$$

with $v$ given by the solution of the second line of (20), i.e.,

$$
\sum_{k \in \mathbb{K}_{\mathrm{on}}} \log _{2}\left(1+P_{R, \mathrm{DF}}^{(k)}(v) \eta_{R, D}^{(k)}\right)=M \hat{R} T,
$$

where $\hat{R}=\left(R-\tau_{m p}^{*} C_{S, D}\right) /\left(1-\tau_{m p}^{*}\right)$, and $C_{S, D}=1 /(M T)$ $\sum_{k \in \mathbb{K}_{\mathrm{on}}} \log _{2}\left(1+P_{S, D F}^{(k)} \gamma_{S, D}^{(k)}\right)$.
The power needed by the DF mode to reach the rate $R$ under the PSD constraint $\bar{P}$ is

$$
P_{\mathrm{DF}}=\tau_{m p}^{*} P_{S, \mathrm{DF}}+\left(1-\tau_{m p}^{*}\right) P_{R, \mathrm{DF}} .
$$

Therefore, we solve (8) using (11) and (23). Clearly, there are cases where a solution to the power minimization problem under a target rate and a PSD constraint does not exist. In particular, when only DT or DF admits a solution, the algorithm will choose the mode for which the solution exists. When the solution does not exist for both DT and DF, the algorithm will choose the mode that achieves the highest rate.

Finally, from (11), (18), and (21) we note that the power allocation for the source node in both DT and DF modes, and for the relay node in DF mode, follows a typical waterfilling shape, where the maximum allowable power in each sub-channel is limited by the power constraint. Therefore, to solve $\{(11),(13)\},\{(18),(19)\},\{(21)$, and (22) $\}$ we can use conventional algorithms for solving power allocation problems when a sub-channel power constraint is imposed [25].

\section{Opportunistic Amplify and Forward}

In order to compare the performance of ODF with a simpler relay scheme, in "Numerical results" section, we also consider OAF.

For clarity, in the following, we describe the essence of the protocol.

Assuming the system model of "PLC system model" section, the achievable rate of OAF can be computed as

$$
C_{\mathrm{OAF}}=\max \left\{C_{\mathrm{DT}}, C_{\mathrm{AF}}\right\},
$$

where $C_{\mathrm{DT}}$ is given by (3). The achievable rate of $\mathrm{AF}$ can be computed as follows. We assume a frame normalized duration $T_{f}=1$ and further, we assume that the 
relay amplifies the signal received in sub-channel $k$ by the quantity

$$
g^{(k)}=\sqrt{\frac{P_{R, \mathrm{AF}}^{(k)}}{P_{S, \mathrm{AF}}^{(k)}\left|H_{S, R}^{(k)}\right|^{2}+P_{w, R}^{(k)}}}
$$

to assure that the relay transmits the power $P_{R, \mathrm{AF}}^{(k)}$ in subchannel $k$ during the second half of the time frame. Finally, we assume the receiver adopts maximal ratio combining for the data received from the source and the relay in the two time slots. Therefore, the AF achievable rate can be written as $[5,7,29]$

$$
\begin{aligned}
& C_{\mathrm{AF}}\left(\mathbf{P}_{\mathbf{S}, \mathbf{A F}}, \mathbf{P}_{\mathbf{R}, \mathrm{AF}}\right)=\frac{1}{2 M T} \\
& \quad \times \sum_{k \in \mathbb{K}_{\mathrm{on}}} \log _{2}\left(1+P_{S, \mathrm{AF}}^{(k)} \eta_{S, D}^{(k)}+\frac{P_{S, \mathrm{AF}}^{(k)} \eta_{S, R}^{(k)} P_{R, \mathrm{AF}}^{(k)} \eta_{R, D}^{(k)}}{1+P_{S, \mathrm{AF}}^{(k)} \eta_{S, R}^{(k)}+P_{R, \mathrm{AF}}^{(k)} \eta_{R, D}^{(k)}}\right) .
\end{aligned}
$$

From (26), we note that the second and the third arguments of the log function, respectively, denote the SNR obtained with the direct link, and the one obtained with the relay. Furthermore, the term 1/2 accounts for the slot duration. Clearly, the second term is null when the source cannot directly reach the destination.

\section{Rate improvements with OAF}

Looking at (24), we see that the use of OAF can bring achievable rate improvements w.r.t. DT. To maximize the achievable rate of OAF we need to optimally allocate the power for both DT and AF modes. As explained in "Rate improvements with ODF" section, assuming that the network nodes have to satisfy a PSD mask constraint, the sub-channel power allocation that maximizes the DT capacity corresponds to the one given by the same PSD constraint, namely, the DT capacity is maximized when $P_{S, \mathrm{DT}}^{(k)}=\bar{P}, \forall k \in \mathbb{K}_{\mathrm{on}}$.

To maximize the achievable rate of AF subject to a PSD constraint, we notice that (26) is sum of monotonic increasing functions of both the power at the source and at the relay node. Therefore, since we have a constraint on the PSD, we can assert that the optimal power allocation is equal to that given by the same PSD, i.e., $P_{x, \mathrm{AF}}^{(k)}=\bar{P}$, with $x \in\{S, R\}$, and $k \in \mathbb{K}_{\text {on }}$.

Eventually, to compute the OAF achievable rate when the system is subject to a PSD constraint, we can simply compute the DT and the AF achievable rates obtained setting the powers to $\bar{P}$, and then we can choose the mode that gives the highest achievable rate (24).

\section{Power saving with OAF}

In "Power saving with ODF" section, we have explained why the use of ODF can potentially bring to power saving w.r.t. the DT. For the same reasons, also OAF can potentially bring to power saving w.r.t. DT.

To compute the power used by OAF when the communication is subjected to a target rate $R$ constraint and to a PSD constraint, we can solve the problem

$$
P_{\mathrm{OAF}}=\min \left\{P_{\mathrm{DT}}, P_{A F}\right\}
$$

where $P_{\mathrm{DT}}$ and $P_{\mathrm{AF}}$, respectively, denote the minimum power required by the $\mathrm{DT}$ and the AF modes to achieve a rate $R$ under a PSD constraint. The optimal power allocation for DT can be found as in (9), whereas $P_{\mathrm{AF}}$ is the solution $^{\mathrm{a}}$ to the problem

$$
P_{\mathrm{AF}}=\min \frac{1}{2} \sum_{k \in \mathbb{K}_{\mathrm{on}}}\left(P_{S, \mathrm{AF}}^{(k)}+P_{R, \mathrm{AF}}^{(k)}\right)
$$

s.t. $C_{\mathrm{AF}}=R$,

$$
0 \leq P_{S, \mathrm{AF}}^{(k)} \leq \bar{P}
$$$$
0 \leq P_{R, \mathrm{AF}}^{(k)} \leq \bar{P}, \quad \forall \quad k \in \mathbb{K}_{\mathrm{on}} .
$$

Problem (28) is not convex because the equality constraint is not an affine function of the transmitted powers. We further report that we have not found a way to reduce the problem to an equivalent convex optimization problem. Therefore, when showing numerical results, we solve (28) using the interior-point method [26, chapter 11].

\section{Numerical results}

In this section, we analyze the performance of ODF and OAF protocols for rate improvements, power saving, and coverage extension over in-home PLC networks. To this end, we first describe, in "Multi-carrier system parameters" section, the OFDM system parameters. Then, in "Experimental channels" section, we describe the sites where the experimental channels have been measured. Since channel measurements were limited to pairs of outlets, to investigate performance with the relay placed in the MP or in derivation boxes and to allow for more general network topologies, we also consider the use of a statistical channel generator in "Statistical channel generator" section. Numerical results are finally presented and discussed in "Achievable rate improvements with ODF and OAF" and "Power saving with ODF and OAF" sections.

\section{Multi-carrier system parameters}

We consider a multi-carrier scheme with parameters similar to those employed by the HomePlug AV broadband PLC system [30]. The system uses $M=1536$ sub-channels in the frequency band $0-37.5 \mathrm{MHz}$, unless otherwise stated. The set $\mathbb{K}_{\text {on }}$ of sub-channels that are switched on 
is defined so that the transmission band is $1-28 \mathrm{MHz}$. To respect the EMC norms [31], we consider the PSD mask constraint of $-50 \mathrm{dBm} / \mathrm{Hz}$. Furthermore, we assume that the relay and the destination nodes experience white Gaussian noise both with PSD equal to $-110 \mathrm{dBm} / \mathrm{Hz}$ (worst case) or $-140 \mathrm{dBm} / \mathrm{Hz}$ (best case).

\section{Experimental channels}

The experimental channels have been measured in two sites. The first site is representative of a single-subtopology network. It is an urban flat of $100 \mathrm{~m}^{2}$ that comprises 6 rooms where the outlets are fed by the same $\mathrm{CB}$ and a total amount of 13 outlets is present. The second site is representative of a two-sub-topology network (see Figure 3). It is a two-floor detached house of $170 \mathrm{~m}^{2}$ composed of 9 rooms, and two electrical circuits, one for each sub-topology, that are connected at the MP each with a CB. The number of accessible outlets is 24 (13 in one floor and 11 in the other). In the first site, the channels have been measured between all pairs of outlets. These channels will be used to analyze the ORA configuration (see "In-home power line network topology" section). In the second site, only the channels between pairs of outlets belonging to different sub-topologies were measured. These channels will be used to analyze the ORAS and ORAD configurations. Details regarding the measurement setup are reported in [32].

\section{Statistical channel generator}

According to experimental evidence and norms, statistically representative in-home power line channel generators have been developed in $[17,18]$. In the following, we briefly describe the channel generator presented in $[17,18]$, which is representative of EU topologies. It uses a statistical topology model together with the computation of the channel responses through the application of transmission line theory. To be more precise, a location plan with a given area $A_{f}$ contains $N_{c}=\left\lceil A_{f} / A_{c}\right\rceil$ clusters of area $A_{c}$ (see Figure 3 ). The outlets are distributed only along the cluster perimeter. The number of outlets belonging to a given cluster is modeled as a Poisson variable with intensity $\Lambda_{o} A_{c}$. Furthermore, the outlets are uniformly distributed along the perimeter. The impact of the loads is also taken into account. In particular, a set of $N_{L}=20$ measured loads for the in-home scenario, such as lamps or computer transformers, is considered. The loads characterization has been done as reported in ([33], Sec. 2.5.2). Furthermore, the impedance of the $S, D$, and $R$ nodes is set to $50 \Omega$.

To generate network topologies, we assume the home and the cluster areas equal to $A_{f}=200 \mathrm{~m}^{2}$ and $A_{c}=20 \mathrm{~m}^{2}$, for the single sub-topology networks, whereas, $A_{f}=300 \mathrm{~m}^{2}$, for the two-sub-topology networks. We set the probability that no load is connected to a given outlet to 0.3 . The intensity $\Lambda_{o}$ is set to 0.33 (outlets $/ \mathrm{m}^{2}$ ). Furthermore, for the two-sub-topology networks, we model each CB with a frequency attenuation, obtained from experimental measurements, that monotonically decreases from about -0.1 to $-3.8 \mathrm{~dB}$ in the $1-28 \mathrm{MHz}$ band.

More details regarding the network topology generator can be found in [18]. The simulator uses larger areas than those corresponding to the measured channels to allow investigating whether higher gains are attainable with the use of the relay. Finally, unless otherwise stated, when showing results over generated channels, we consider 100 network topologies. For each network topology, we consider 10 pairs of outlets (links S-D), and for each pair of randomly picked outlets, we place the relay according to the configurations presented in "In-home power line network topology" section.

\section{Achievable rate improvements with ODF and OAF}

In Table 1, we report the average achievable rate values obtained using DT and ODF for both noise levels over the experimental channels for the ORA (site 1), and ORAS, ORAD configurations (site 2). The percentile usage of the relay is also reported. Table 1 also reports the results for OAF. These will be discussed later. As we can see from Table 1, the use of the relay for the considered configurations increases the mean achievable rate w.r.t. the DT by $3,4.2$, and $6.6 \%$, respectively, for the ORA, ORAS, and ORAD configurations with noise PSD equal to $-110 \mathrm{dBm} / \mathrm{Hz}$. The gains are around the $1 \%$ for the low noise level case. The improvements are not very high and this is due to the small area of the experimental sites, which leads to moderate channel attenuations. Although not reported, we have found comparable results using the statistical channel generator with parameters similar to the two experimental sites. Furthermore, we notice that experimental results did not allow for investigating the effect of placing the relay in other locations as the MP and derivation boxes.

To infer performance with the use of the relay in other positions-namely, in the MP and in the derivation boxes-and to consider larger areas, we discuss the numerical results obtained using the statistical channel generator. Table 2 lists the average achievable rate values considering all the strategic relay configurations presented in the "In-home power line network topology" section for both noise levels. Furthermore, Figure 5 shows the complementary cumulative distribution function (CCDF) of the achievable rate for DT mode, when no relay is connected to the network, and for the ODF protocol according to the various relay configurations in a single-sub-topology network. The noise PSD is set to $-110 \mathrm{dBm} / \mathrm{Hz}$. All the previous results are obtained computing the time slot 
Table 1 Average achievable rate values using ODF and OAF for the various relay configurations over the measured channels

\begin{tabular}{|c|c|c|c|c|c|c|c|c|}
\hline \multirow[b]{2}{*}{$\begin{array}{l}\text { Sub } \\
\text { topology }\end{array}$} & \multirow[b]{2}{*}{ Conf. } & \multirow[b]{2}{*}{$\begin{array}{c}C_{S, D} \\
(\mathrm{Mbit} / \mathrm{s})\end{array}$} & \multicolumn{3}{|c|}{ ODF } & \multicolumn{3}{|c|}{ OAF } \\
\hline & & & $\begin{array}{c}C_{\mathrm{DF}} \\
(\mathrm{Mbit} / \mathrm{s})\end{array}$ & $\begin{array}{c}C_{\mathrm{ODF}} \\
(\mathrm{Mbit} / \mathrm{s})\end{array}$ & $\begin{array}{c}\% \text { use of } \\
\text { relay }\end{array}$ & $\begin{array}{c}C_{\mathrm{AF}} \\
(\mathrm{Mbit} / \mathrm{s})\end{array}$ & $\begin{array}{c}C_{\mathrm{OAF}} \\
(\mathrm{Mbit} / \mathrm{s})\end{array}$ & $\begin{array}{c}\% \text { use of } \\
\text { relay }\end{array}$ \\
\hline \multicolumn{9}{|c|}{ Noise PSD $=-110(\mathbf{d B m} / \mathbf{H z})$} \\
\hline Single & ORA & 170.8 & 63 & 176 & 30.3 & 96 & 171 & 2.3 \\
\hline \multirow{2}{*}{ Two } & ORAS & 136.4 & 66.6 & 142.2 & 20 & 80.4 & 136.7 & 2.1 \\
\hline & ORAD & 142.5 & 67.5 & 151.9 & 23.9 & 88.7 & 142.8 & 2.9 \\
\hline \multicolumn{9}{|c|}{ Noise PSD $=-140(\mathbf{d B m} / \mathbf{H z})$} \\
\hline Single & ORA & 428.9 & 193.2 & 432.9 & 36 & 228.4 & 428.9 & 0 \\
\hline \multirow{2}{*}{ Two } & ORAS & 396.8 & 201 & 400.7 & 34.6 & 213.4 & 396.8 & 0 \\
\hline & ORAD & 403.3 & 193.6 & 409.7 & 46.9 & 282.1 & 403.3 & 0 \\
\hline
\end{tabular}

$\tau$ according to (7), i.e., $\tau=\tau_{m r}^{*}$. From Table 2, we notice that the average capacity values, for the link $\mathrm{S}-\mathrm{D}$, vary with the relay configuration. This is because the electrical properties of the network depend on the relay placement, which is different in each configuration.
From Figure 5 and Table 2, we notice that in general there are two relay configurations for which we obtain high achievable rate gains with ODF and these are the SDB and the BDB. With probability equal to 0.8 , these allow for an achievable rate improvement, w.r.t. DT, of about $50 \%$.

Table 2 Average achievable rate values using ODF and OAF for the various relay configurations over the generated channels

\begin{tabular}{|c|c|c|c|c|c|c|c|c|}
\hline \multirow[b]{2}{*}{$\begin{array}{l}\text { Sub } \\
\text { topology }\end{array}$} & \multirow[b]{2}{*}{ Conf. } & \multirow[b]{2}{*}{$\begin{array}{c}C_{S, D} \\
(M b i t / s)\end{array}$} & \multicolumn{3}{|c|}{ ODF } & \multicolumn{3}{|c|}{ OAF } \\
\hline & & & $\begin{array}{c}C_{\mathrm{DF}} \\
\text { (Mbit/s) }\end{array}$ & $\begin{array}{c}C_{\mathrm{ODF}} \\
\text { (Mbit/s) }\end{array}$ & $\begin{array}{c}\% \text { use of } \\
\text { relay }\end{array}$ & $\begin{array}{c}C_{\mathrm{AF}} \\
(\mathrm{Mbit} / \mathrm{s})\end{array}$ & $\begin{array}{c}C_{\mathrm{OAF}} \\
(\mathrm{Mbit} / \mathrm{s})\end{array}$ & $\begin{array}{c}\% \text { use of } \\
\text { relay }\end{array}$ \\
\hline \multicolumn{9}{|c|}{ Noise PSD $=-110(\mathbf{d B m} / \mathrm{Hz})$} \\
\hline \multirow[t]{6}{*}{ Single } & SDB & 182.8 & 220.2 & 220.2 & 99.9 & 128.9 & 183.8 & 12 \\
\hline & $\mathrm{BDB}$ & 183.2 & 216.5 & 216.8 & 99.9 & 127 & 186.4 & 18.8 \\
\hline & $\mathrm{RDB}$ & 190.3 & 104.3 & 207.6 & 52.8 & 113.9 & 191.9 & 11.1 \\
\hline & MPS & 190.2 & 99.2 & 205.9 & 48.5 & 112.5 & 191.7 & 9.5 \\
\hline & ORA & 193.6 & 70.6 & 202.7 & 29.8 & 107.7 & 194.2 & 6.6 \\
\hline & DDB & 182.9 & 189 & 189.9 & 99.4 & 106.2 & 183 & 0.7 \\
\hline \multirow[t]{3}{*}{ Two } & MPM & 116.4 & 131.5 & 148.6 & 91.3 & 87.6 & 120.2 & 28.6 \\
\hline & ORAS & 121.2 & 73.2 & 128.2 & 33.8 & 71 & 122 & 7.5 \\
\hline & ORAD & 121.3 & 51.8 & 127.9 & 31.5 & 70.7 & 121.9 & 6.1 \\
\hline \multicolumn{9}{|c|}{ Noise PSD $=-140(\mathbf{d B m} / \mathbf{H z})$} \\
\hline \multirow[t]{6}{*}{ Single } & SDB & 421 & 454.8 & 454.9 & 99.9 & 255.7 & 421 & 0.3 \\
\hline & $\mathrm{BDB}$ & 421.3 & 453.3 & 453.9 & 99.9 & 255.7 & 422 & 3.5 \\
\hline & $\mathrm{RDB}$ & 429.4 & 238.8 & 447.6 & 53 & 240.4 & 430 & 2.3 \\
\hline & MPS & 429.3 & 223.8 & 445 & 48.1 & 237.7 & 429.8 & 1.6 \\
\hline & ORA & 433.8 & 181.3 & 444.7 & 32.5 & 233.7 & 434 & 1 \\
\hline & DDB & 420.9 & 426.2 & 427.6 & 99.6 & 228.3 & 420.9 & 0 \\
\hline \multirow[t]{3}{*}{ Two } & MPM & 341 & 339 & 376 & 91.7 & 215.6 & 342.3 & 5 \\
\hline & ORAS & 348 & 232.8 & 357 & 38.4 & 191.9 & 348.3 & 1.5 \\
\hline & ORAD & 348 & 161.7 & 356.4 & 40.1 & 191.2 & 348.2 & 1.2 \\
\hline
\end{tabular}




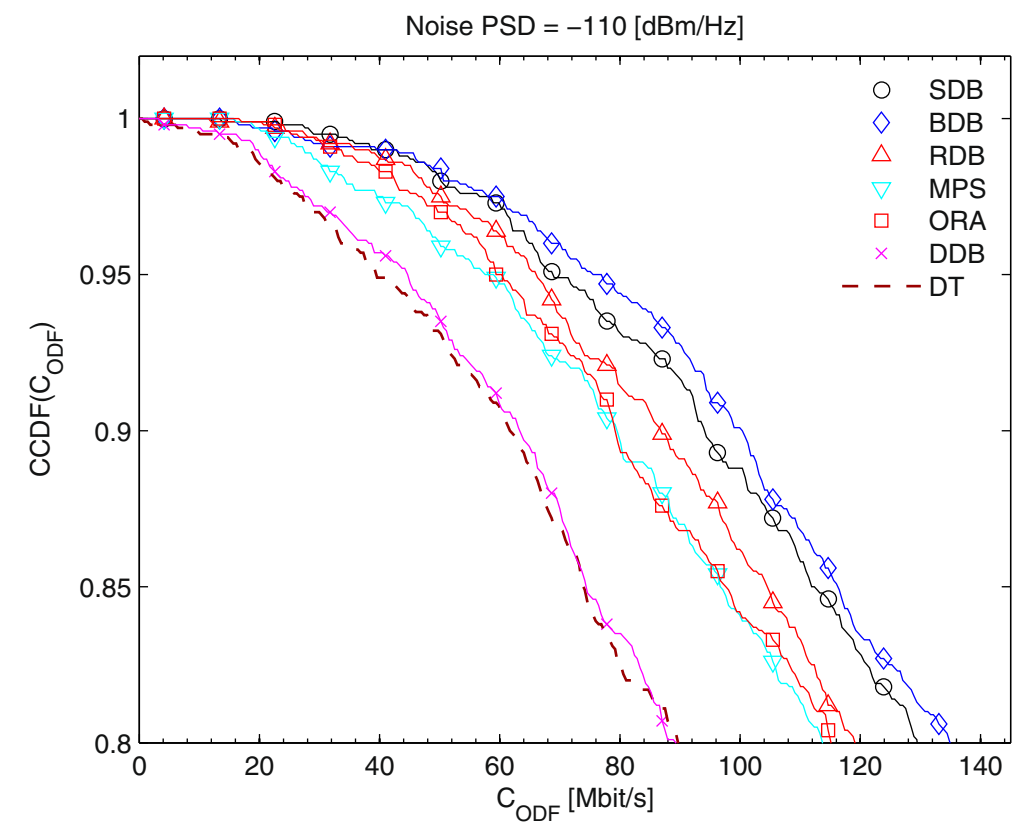

Figure 5 CCDF of achievable rate obtained using ODF with the relay located according to the various described configurations in single-sub-topology networks. The CCDF of capacity obtained assuming DT mode when no relay is connected to the network is also reported $M=1536$.

From Table 2, we notice that, as obtained over experimental channels, also over generated channels, the ORA configuration gives small achievable rate improvements w.r.t. the DT. From Figure 5, the relay positions can be ordered from worst to best (at CCDF $=0.8$ ) as DDB, MPS, ORA, RDB, SDB, BDB. Although not shown, the same applies for the low noise scenario, where we found that the SDB gain, w.r.t. the DT, equals $20 \%$. The average rates are reported in Table 2, according to which the relay positions can be ordered from worst to best as DDB, ORA, MPS, RDB, BDB, SDB.

Now, considering the two-sub-topology case, from Table 2, we notice that the MPM relay configuration gives the best performance. In particular, it gives an achievable rate improvement of 27 and 10\%, respectively, for the high and for the low noise level. Another important observation regards the percentile usage of the relay for the various configurations. As explained in "Opportunistic Decode and Forward" section, the necessary condition not to use the direct link is $C_{S, D}<\min \left\{C_{S, R}, C_{R, D}\right\}$. This condition is satisfied when the relay lies on the backbone between the source and the destination nodes, which is always true for the SDB, BDB, DDB, and MPM relay configurations. Therefore, these configurations are also the ones for which the relay is mostly used (see Table 2). We further note that as shown in "Rate improvements with ODF" and "Rate improvements with OAF" sections, the power allocation that maximizes the DT and the DF achievable rates is given by the PSD limit level. Therefore, all the curves of Figure 5, and all the results of Table 2, are obtained on equal transmitted power.

We now turn our attention to the achievable rate improvements provided by the OAF protocol. Figure 6 shows the CCDFs of the achievable rate. From Figure 6, we note that in general OAF does not bring appreciable achievable rate improvements w.r.t. DT. In particular, we notice that the best relay position, in terms of reliability, namely, minimum rate value, is the SDB. With probability equal to 0.8 , it assures an achievable rate gain, w.r.t. the DT, equal to 5.6 and $0 \%$, respectively, for the high and the low noise levels (although the low noise level results are not explicitly shown). This result agrees with what is reported in [34] where it is shown that in low SNR wireless scenarios, the AF protocol does not perform well. This is because the noise is also amplified at the relay. From Figure 6, we finally note that there are some OAF relay configurations that give worse performance than the DT. This is because the curve labeled with DT is obtained when no relay is connected to the network, whereas, the other curves are obtained with the relay connected to the network, therefore, the electrical network properties are different for the various configurations. It is interesting to note that the SDB relay configuration is not the one that give the best performance in terms of average achievable rate. In fact, looking at Table 2, we can see that the relay configuration that yields the highest average achievable rate for OAF is the ORA. 


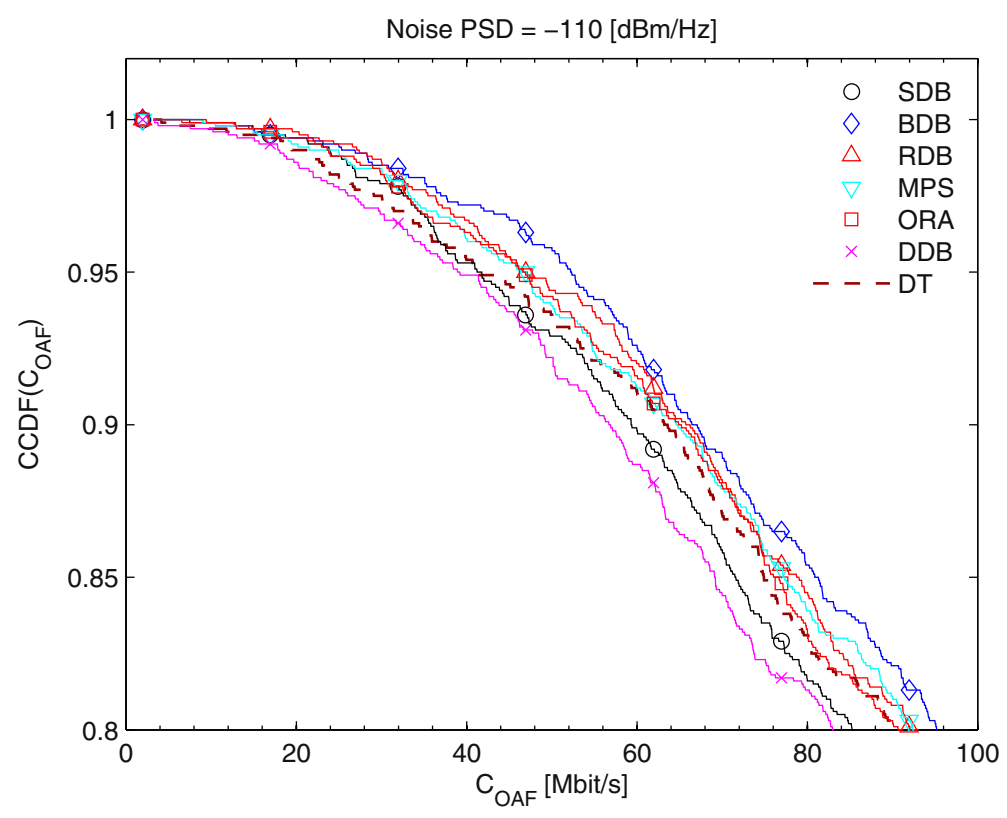

Figure $6 \mathrm{CCDF}$ of achievable rate obtained using OAF with the relay located according to the various described configurations in single-sub-topology networks. The CCDF of capacity obtained assuming DT mode when no relay is connected to the network is also reported $M=1536$.

From the previous results we highlight that, over the considered in-home power line network topologies (see "In-home power line network topology" section), ODF gives good rate improvements w.r.t. DT over both single and two sub-topology networks, whereas OAF does not bring any substantial benefit. Furthermore, in ODF, the gains are more significant when the relay is placed in a backbone node.

\section{Power saving with ODF and OAF}

Figure 7 shows the power required by DF to achieve a target rate $R$ equal to $20 \mathrm{Mbit} / \mathrm{s}$ when using the optimal and the simplified DF power allocation algorithm (see "Optimal DF power allocation" and "Simplified algorithm for DF power allocation" sections). Due to the high computational complexity of the optimal solution, we only show the results for ten network realizations, and for $M=62$ sub-channels. The time slot duration is set equal to $t_{m r}^{*}$. The noise PSD is set to $-140 \mathrm{dBm} / \mathrm{Hz}$. The considered relay configuration is the MPM. The optimal solution is obtained considering the interior-point algorithm using as the starting point either the power distribution given by the PSD mask, and the solution of the simplified algorithm. From Figure 7, we note that the proposed DF power allocation algorithm gives results that are almost equal to the optimal ones. Furthermore, we highlight that the cases where the proposed algorithm performs slightly better than the optimal one are due to the numerical precision of the simulation. Therefore, from now on, we only show results for ODF obtained using the proposed DF power allocation algorithm.

In order to assess the performance of the proposed ODF power allocation algorithm (see "Simplified algorithm for DF power allocation" section), we set the target rate equal to the capacity of the DT link, i.e., $R=C_{\mathrm{DT}}$ when $P_{S, \mathrm{DT}}^{(k)}=\bar{P} \forall k \in \mathbb{K}_{\text {on }}$ (see also Figure 5 ). Figure 8 shows the cumulative distribution function (CDF) of the total transmitted power for DT, and for ODF (8) when considering the various single-sub-topology relay configurations. The noise level is set to $-110 \mathrm{dBm} / \mathrm{Hz}$. From Figure 8 , we can see that the best relay position is the SDB. With probability equal to 0.8 , it allows for saving $2.6 \mathrm{~dB}$. With the same probability, the BDB relay configuration allows for saving about $1.2 \mathrm{~dB}$. We have found that similar results are obtained considering the low noise level. In particular, with probability equal to 0.8 , the SDB and the BDB configurations lead to a power saving of 2 and $0.9 \mathrm{~dB}$.

In the right sub-plot of Figure 9, we show the results the for DT mode, and for the OAF mode. Also in this case, the noise level is set to $-110 \mathrm{dBm} / \mathrm{Hz}$. The results obtained using ODF (Figure 9left) are also reported. For fairness of comparison, we set for both schemes $M=62$, and we consider 100 network realizations. From Figure 9, we note that the best relay configurations for power saving with OAF is the BDB. From Figure 9, we also notice that ODF yields higher power saving than OAF. Finally, it is interesting to note that the behavior of the performance of 


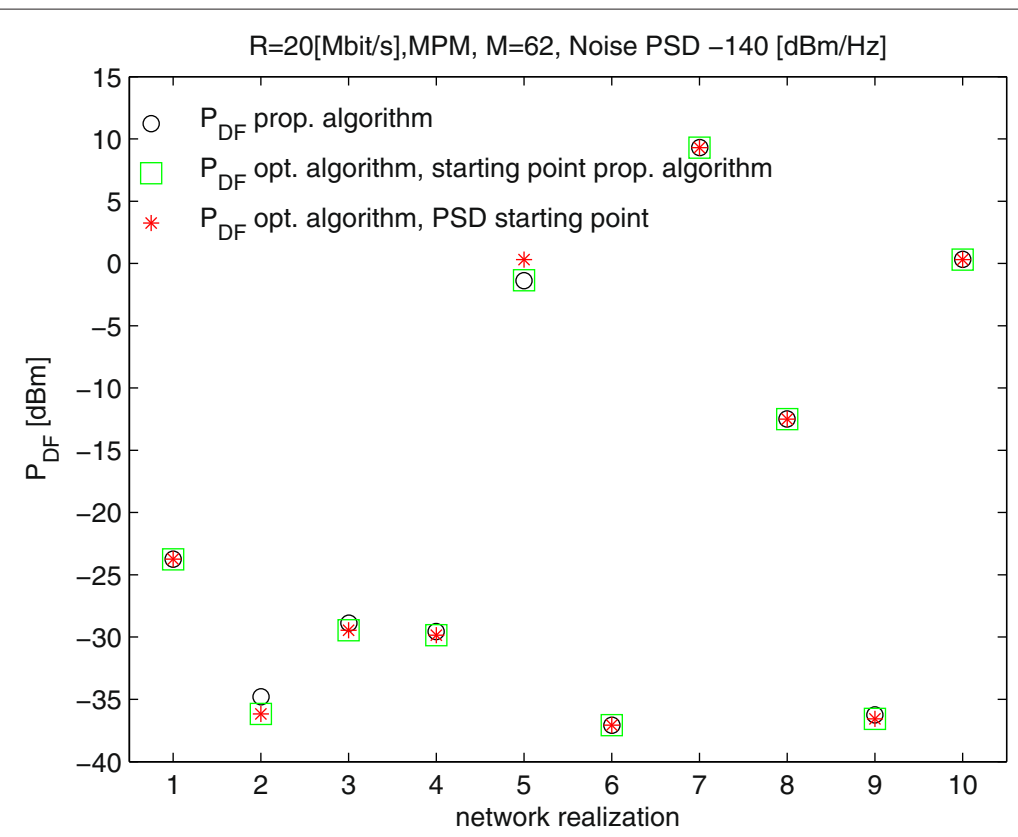

Figure 7 Power required by ODF to achieve a target rate $R$ equal to $20 \mathrm{Mbit} / \mathrm{s}$ when using the optimal DF power allocation algorithm and the simplified algorithm. The optimal solution is obtained considering the interior-point algorithms starting from two points, i.e., the power distribution given by the PSD mask and the solution of the simplified algorithm.

ODF does not appreciably depend on the number of subchannels $M$, i.e., the ranking of the relay positions does not change for the two values of $M$ (compare Figures 8 and 9 left).
We now turn our attention to the network coverage (number of links satisfying the target rate) improvements that can be obtained with the use of a relay. To this end, we consider the MPM, the ORAS, and the ORAD relay

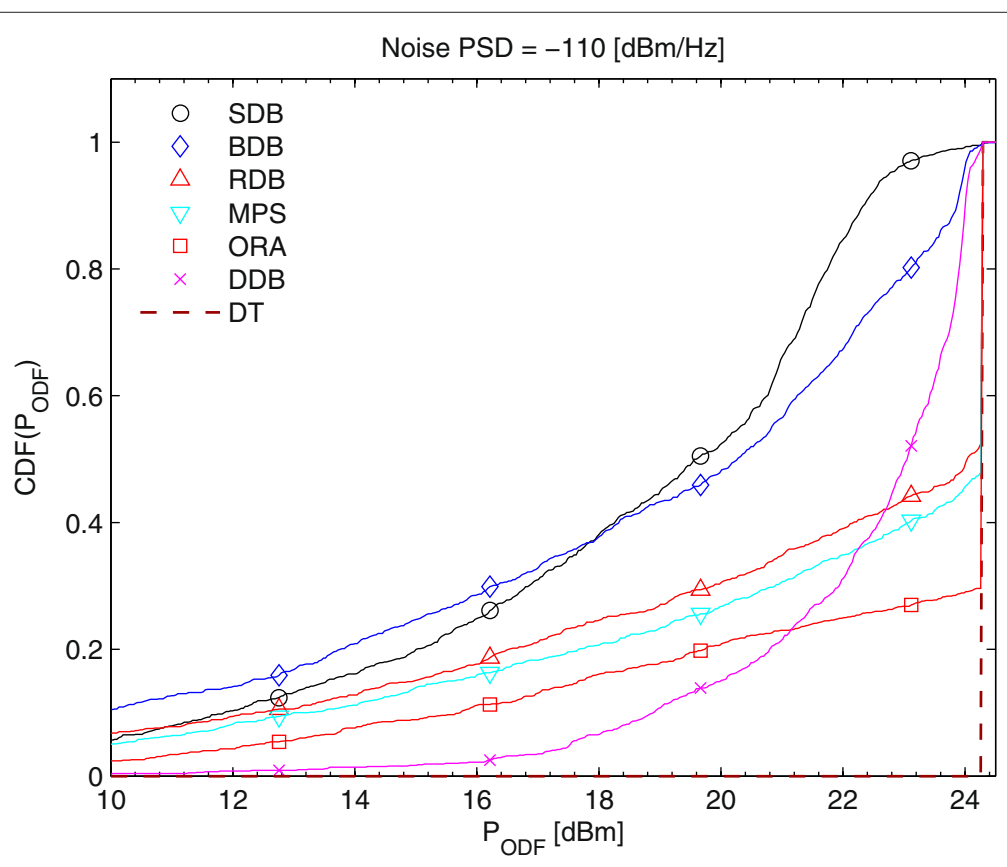

Figure $8 \mathrm{CDF}$ of the total transmitted power using ODF with the relay located according to the various described configurations in single-sub-topology networks, and the DT $M=1536$. 

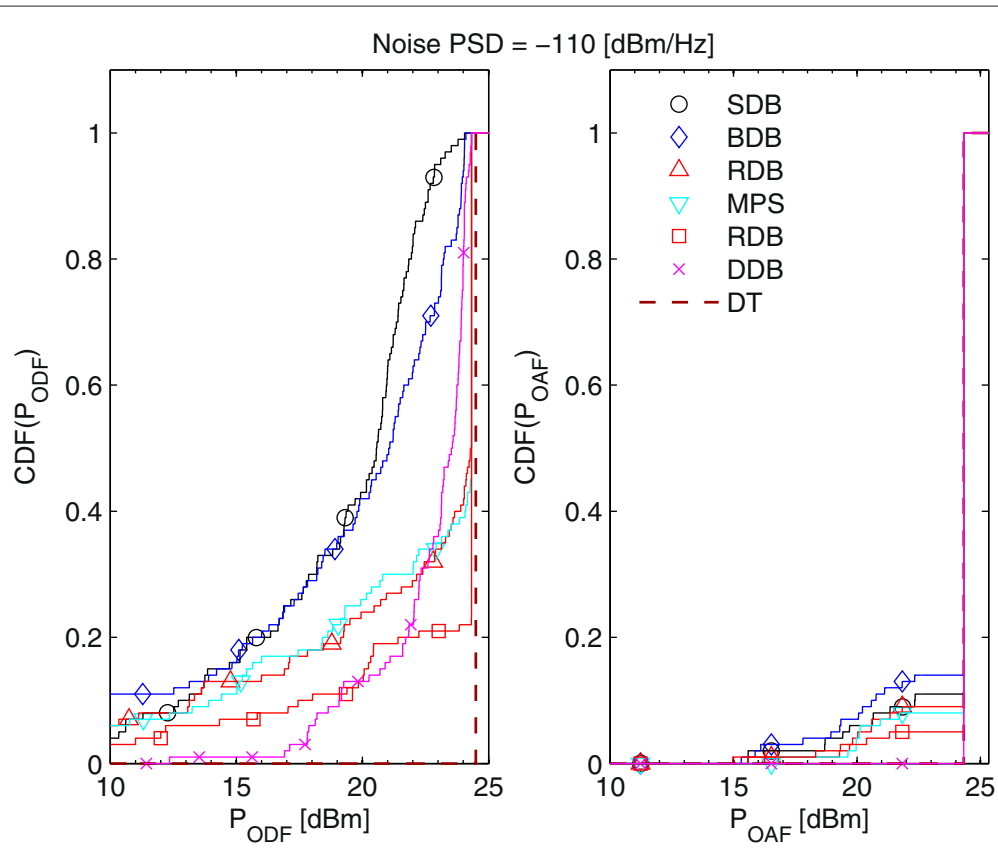

Figure $9 \mathrm{CDF}$ of the total transmitted power using (left) ODF and (right) OAF with the relay located according to the various described configurations in single-sub-topology networks $M=62$.

configurations in a two-sub-topology network. Since the $\mathrm{S}-\mathrm{D}$ links experience high attenuation given by the presence of CBs in the MP, we infer that the use of the relay should yield high coverage extension. To validate our conjecture we consider the following scenario. We impose a target rate of $100 \mathrm{Mbit} / \mathrm{s}$, e.g., as required by a multimedia application to be delivered from the living room to the bedroom located in an upper floor. In Table 3, we report the percentage of links that satisfy the requirements for both measured and generated channels using DT, and ODF with MPM, ORAS, and ORAD relay configurations.
We also report the average total transmitted power. Looking at the results, we note that the use of the relay does not substantially increase the coverage when we place it in a random outlet belonging to either the source or the destination sub-topology (ORAS and ORAD configurations). Notably, when we place the relay in between the CBs of the MP (MPM configuration), for the high noise level, the coverage increases by $47 \%$ and the corresponding power saving is equal to $1.9 \mathrm{~dB}$. When the noise level is low, we still have high power saving given by the use of a relay, but the gains associated

Table 3 Percentage of satisfied links and mean transmitted power for two-sub-topology networks using DT and ODF for the measured and generated channels (the rate target is $100 \mathrm{Mbit} / \mathrm{s}$ )

\begin{tabular}{|c|c|c|c|c|c|c|c|c|}
\hline \multirow{4}{*}{ Conf. } & \multicolumn{4}{|c|}{ Measured channels (area $170 \mathrm{~m}^{2}$ ) } & \multicolumn{4}{|c|}{ Generated channels (area $300 \mathrm{~m}^{2}$ ) } \\
\hline & \multicolumn{2}{|c|}{ DT } & \multicolumn{2}{|c|}{ ODF } & \multicolumn{2}{|c|}{ DT } & \multicolumn{2}{|c|}{ ODF } \\
\hline & $\begin{array}{c}\% \text { of } \\
\text { satisfied }\end{array}$ & $E\left[P_{\mathrm{DT}}\right]$ & $\begin{array}{c}\% \text { of } \\
\text { satisfied }\end{array}$ & $E\left[P_{\mathrm{ODF}}\right]$ & $\begin{array}{c}\% \text { of } \\
\text { satisfied }\end{array}$ & $E\left[P_{\mathrm{DT}}\right]$ & $\begin{array}{c}\% \text { of } \\
\text { satisfied }\end{array}$ & $E\left[P_{\mathrm{ODF}}\right]$ \\
\hline & links & $(\mathrm{dBm})$ & links & $(\mathrm{dBm})$ & links & $(\mathrm{dBm})$ & links & $(\mathrm{dBm})$ \\
\hline \multicolumn{9}{|c|}{ Noise PSD $=-110(\mathbf{d B m} / \mathbf{H z})$} \\
\hline ORAS & 75 & 21.2 & 79.6 & 20.9 & 54.9 & 21.8 & 59.3 & 21.5 \\
\hline ORAD & 76.9 & 21 & 82 & 20.3 & 55.2 & 21.8 & 59.1 & 21.5 \\
\hline MPM & - & - & - & - & 52.4 & 22 & 77 & 20.1 \\
\hline \multicolumn{9}{|c|}{ Noise PSD $=-140(\mathbf{d B m} / \mathbf{H z})$} \\
\hline ORAS & 100 & -5.8 & 100 & -5.9 & 97.9 & 9.5 & 99 & 7.2 \\
\hline ORAD & 100 & -6.1 & 100 & -6.4 & 97.9 & 9.5 & 98.7 & 7.6 \\
\hline MPM & - & - & - & - & 97.8 & 9.8 & 99.9 & 2.6 \\
\hline
\end{tabular}


to the coverage extension are reduced below 5\%. This is simply explainable observing that for low noise levels the imposed target rates are also achievable using the DT.

Regarding the OAF, we notice that it does not bring to any appreciable power saving and/or coverage increase.

\section{Conclusions}

We have investigated the use of half duplex time division ODF and OAF relay protocols to provide achievable rate improvements, power saving, and coverage extension over in-home PLC networks.

We have found the optimal power and time slot allocation that maximizes the ODF achievable rate with a PSD constraint. Furthermore, considering the power minimization problem of ODF with a target rate constraint, since the optimal solution involves a complex procedure, we have proposed a simplified algorithm that is based on the resolution of two convex sub-problems. Through numerical results, we have shown that its performance is very close to the optimal one.

The specific and peculiar application of the algorithms to the in-home PLC scenario has been considered. The results show that, in general, ODF performs better than OAF. Significant rate improvements and power savings can be obtained depending on the relay position and the size of the network. In the considered single circuit (single sub-topology) network, with high reliability, achievable rate gains (up to $50 \%$ ), or power savings (up to $3 \mathrm{~dB}$ ), are offered by ODF when the relay is placed in the derivation box that feeds the source node or in a derivation box that lies on the backbone link between the source and the destination nodes. In the considered two circuits network connected at the MP, e.g., a typical two-floor house network, the best relay location is in the MP. Also in this case, substantial achievable rate improvements (up to $27 \%$ ), power savings (up to $1.9 \mathrm{~dB}$ ) and coverage extension (up to $47 \%$ ) have been found.

\section{Endnote}

${ }^{\text {a}}$ Note that in some cases it is possible that the target rate is not reachable under a PSD constraint and thus the power minimization problem does not admit a solution.

\section{Competing interests}

The authors declare that they have no competing interests.

Received: 28 May 2012 Accepted: 14 August 2012

Published: 5 September 2012

\section{References}

1. AM Tonello, S D'Alessandro, L Lampe, Cyclic prefix design and allocation in bit-loaded OFDM over power line communication channels. IEEE Trans. Commun. 58(11), 3265-3276 (2010)

2. A Host-Madsen, J Zhang, Capacity bounds and power allocation for the wireless relay channel. IEEE Trans. Inf. Theory. 51 (6), 2020-2040 (2005)
3. D Gündüz, E Erkip, Opportunistic cooperation by dynamic resource allocation. IEEE Trans. Wirel. Commun. 6(4), 1446-1454 (2007)

4. L Xie, X Zhang, in Proc. of IEEE Wireless Communications and Networking Conference (WCNC 2007). TDMA and FDMA based resource allocations for quality of service provisioning over wireless relay networks, Hong Kong, China, 2007) pp. 3153-3157

5. I Hammerström, A Wittneben, in Proc. of IEEE International Conference on Communication (ICC 2006), On the optimal power allocation for nonregenerative OFDM relay links. vol. 10 (Istanbul, Turkey, 2006), pp. 4463-4468

6. W Zhang, U Mitra, M Chiang, Optimization of amplify-and-forward multicarrier two-hop transmission. IEEE Trans. Commun. 59(5), 1434-1445 (2011)

7. Y Li, W Wang, J Kong, M Peng, Subcarrier pairing for amplify-and-forward and decode-and-forward OFDM-relay links. IEEE Commun. Lett. 13(4), 209-211 (2009)

8. W Ying, Q Xin-Chun, W Tong, L Bao-Ling, in Proc. of IEEE Vehicular Tech. Conf. (VTC-Spring 2007). Power allocation subcarrier pairing algorithm for regenerative OFDM relay system, Dublin, Ireland, 2007) pp. 2727-2731

9. X Li, J Zhang, J Huang, in Proc. of IEEE Vehicular Tech. Conf. (VTC 2009 Spring). Power allocation for OFDM based links in hybrid forward relay, Barcelona, Spain, 2009)

10. Y Liang, $\vee$ Veeravalli, $\vee$ Poor, Resource allocation for wireless fading relay channels: max-min solution. IEEE Trans. Inf. Theory. 53(10), 3432-3453 (2007)

11. D Gesbert, S Hanly, H Huang, S Shamai-Shitz, W Yu, Guest editorial cooperative communications in MIMO cellular networks. IEEE J. Sel. Areas Commun. 28(9), 1377-1379 (2010)

12. K Bakanoglu, S Tomasin, E Erkip, Resource allocation for the parallel relay channel with multiple relays. IEEE Trans. Wirel. Commun. 10(3), 792-802 (2011)

13. L Lampe, R Shober, S Yiu, Distributed space-time block coding for multihop transmission in power line communication networks. IEEE J. Sel. Areas Commun. 24(7), 1389-1400 (2006)

14. H Zou, A Chowdhery, S Jagannathan, JM Cioffi, JL Masson, in Proc. of IEEE International Conference on Communication (ICC 2009). Multi-user joint subchannel and power resource-allocation for powerline relay networks, Dresden, Germany, 2009)

15. B Tan, J Thompson, in Proc. of IEEE International Conference on Communication (ICC 2011). Relay transmission protocols for in-door powerline communications networks, Kyoto, Japan, 2011)

16. B Tan, J Thompson, in Proc. of European Signal Processing Conference (EUSIPCO 2011). Capacity evaluation with channel estimation error for the decode-and-forward relay PLC networks, EURASIP, Barcelona, Spain, 2011) pp. 834-838

17. AM Tonello, F Versolatto, Bottom-up statistical plc channel modelingPart II: inferring the statistics. IEEE Trans. Power Deliv. 25(4), 2356-2363 (2010)

18. AM Tonello, F Versolatto, Bottom-up statistical plc channel modelingPart I: random topology model and efficient transfer function computation. IEEE Trans. Power Deliv. 26(2), 891-898 (2010)

19. S D'Alessandro, AM Tonello, F Versolatto, in Proc. of IEEE Int. Symp. on Power Line Commun. and Its Appl. (ISPLC 2011). Power savings with opportunistic decode and forward over in-home PLC networks, Udine, Italy, 2011)

20. S D'Alessandro, AM Tonello, L Lampe, Adaptive pulse-shaped OFDM with application to in-home power line communications. Springer Telecommun. Syst. J. 50, 1-11 (2011). doi:10.1007/s11235-010-9410-3

21. Comitato Elettrotecnico Italiano (CEI), Norma CEI per, Impianti Elettrici Utilizzatori-CEI Norm for Electrical Systems. (CEI, Milan, 2007). ISBN 978-88-432-0047-4

22. TM Cover, JA Thomas, Elements of Information Theory. (Wiley \& Sons, New York,2006)

23. H Latchman, R Newman, Int. Sym. on Power Line Communications and Its Applications (ISPLC'07). Speech II. HomePlug Standards for Worldwide Multimedia In-Home Networking and Broadband Powerline Access, (2007). http://www.ieee-isplc.org/2007/docs/keynotes/latchman-newman.pdf

24. S Hanly, D Tse, Multiaccess fading channels-Part, Il: delay-limited capacities. IEEE Trans. Inf. Theory. 44(7), 2816-2831 (1998)

25. N Papandreou, T Antonakopoulos, Bit and power allocation in constrained multi-carrier systems: the single-user case. EURASIP J. Adv Signal Process. 2008(Article ID 643081), 1-14 (2008) 
26. S Boyd, L Vandenberghe, Convex Optimization. (Cambridge University Press, Cambridge, MA,2004)

27. EW Weisstein, Sylvester's criterion. http://mathworld.wolfram.com/ SylvestersCriterion.html

28. HW Kuhn, AW Tucker, in Proc. of Second Berkeley Symp. on Math. Statist. and Prob. Nonlinear programming (California University Press, California, 1951). pp. 481-492

29. G Kramer, I Maric, R Yates, Cooperative communications. Found. Trends Netw., Hanover, MA, USA. 1(3), 271-425 (2006)

30. K Afkhamie, S Katar, L Yonge, R Newman, in Proc. of IEEE Int. Sym. on Power Line Commun. and Its Appl. (ISPLC 2005). An overview of the upcoming HomePlug AV Standard, Vancouver, Canada, 2005) pp. 400-404

31. M Tlich, R Razafferson, G Avril, A Zeddam, in Proc. of IEEE Int. Symp. on Power Line Commun. and its Appl. (ISPLC 2008). Outline about the EMC properties and throughputs of the PLC systems up to $100 \mathrm{MHz}$, Jeju Island, Korea, 2008) pp. 259-262

32. F Versolatto, AM Tonello, in Proc. of IEEE Int. Symp. on Power Line Commun. and its Appl. (ISPLC 2012). On the relation between geometrical distance and channel statistics in in-home PLC networks, Bejing, China, 2012)

33. HC Ferreira, L Lampe, J Newbury, TG Swart, Power Line Communications: Theory and Applications for Narrowband and Broadband Communications over Power Lines. (Wiley \& Sons, New York,2010)

34. J Laneman, D Tse, G Wornell, Cooperative diversity in wireless networks: efficient protocols and outage behavior. IEEE Trans. Inf. Theory. 50(12), 3062-3080 (2004)

Cite this article as: D'Alessandro and Tonello: On rate improvements and power saving with opportunistic relaying in home power line networks. EURASIP Journal on Advances in Signal Processing 2012 2012:194.

\section{Submit your manuscript to a SpringerOpen ${ }^{\circ}$ journal and benefit from:}

- Convenient online submission

- Rigorous peer review

- Immediate publication on acceptance

- Open access: articles freely available online

- High visibility within the field

- Retaining the copyright to your article

Submit your next manuscript at $\boldsymbol{\triangleright}$ springeropen.com 